\section{Exéresis de odontoma complejo mediante osteotomía sagital de rama. Reporte de un caso}

\section{Surgical excision of a complex odontoma via sagittal osteotomy of the mandible. A case report}

\section{Resumen}

El odontoma es un tumor benigno originado a partir de una alteración de células odontogénicas epiteliales y mesenquimatosas diferenciadas con capacidad de formar esmalte, dentina y cemento, siendo la diferencia entre ambos la organización del tejido dentario. La etiología es desconocida pero se ha asociado a infecciones, anomalías hereditarias, hiperactividad odontoblástica y traumatismos. Cuando el odontoma es de tamaño pequeño o promedio, el tratamiento aceptable es la exéresis del tumor. El problema surge con los odontomas grandes, cuya extirpación implica el sacrificio de grandes cantidades de hueso, el potencial de fractura mandibular y el daño del nervio alveolar inferior. Para evitar estas complicaciones en la extirpación de tumores grandes en la mandíbula, Rittersma y van Gool introdujeron en 1979 el uso de la técnica de osteotomía sagital de rama mandibular para tratar esta patología. Se reporta el caso de un paciente varón de 37 años, sin antecedentes sistémicos, que presentó aumento de volumen de hemicara izquierda asociado a dolor y secreción purulenta, se realizó los exámenes clínicos, imagenológico e histopatológicos, llegando al diagnóstico de odontoma complejo infectado. Fue tratado con la técnica osteotomía sagital de rama mandibular exitosamente en el servicio de cirugía bucal y maxilofacial del Hospital Nacional Hipólito Unanue, Lima-Perú.

Palabras clave: Odontoma; Neoplasias mandibulares (fuente: DeCS BIREME).

\section{Caso Clínico}

Nelly Marcelina Carrasco Ortega ${ }^{1, a}$, David Moreno

Villalobos ${ }^{1, a, b}$, Gloria Arellano Porras ${ }^{1, a, b}$, Arturo

Rodríguez Flores ${ }^{1, a, b}$

${ }^{1}$ Hospital Nacional Hipólito Unanue. Lima, Perú.

${ }^{\text {a }}$ Cirujano Dentista.

${ }^{b}$ Cirujano Bucal y Maxilofacial.

\section{Correspondencia:}

Nelly Marcelina Carrasco Ortega

Correo electrónico: nelllymar@hotmail.com

Jirón Enrrique Nerini 1025 - Dpto. 804, torre 2, Lima 1, Perú.

\section{Coautores:}

David Moreno Villalobos

david.moreno.v@hotmail.com

Gloria Arellano Porras

vicky_arellano1603@hotmail.com

Arturo Rodríguez Flores

a.arturor@hotmail.com

\section{Editor invitado:}

Juan Carlos Cuevas-González

Universidad Autónoma de Ciudad Juárez, México.

Conflicto de intereses: los autores declaran no tener conflictos de interés.

Fuente de financiamiento: autofinanciado.

Recibido: 28/06/19

Aceptado: 01/09/19

Publicado: $27 / 11 / 19$ 
no contributory systemic history, who presented increased volume of left side face associated with pain and purulent secretion; clinical, imaging and histopathological examinations were performed, arriving at the diagnosis of infected complex odontoma. It was successfully treated via sagittal osteotomy technique of the mandibular ramus at Hipólito Unanue Hospital's Oral and Maxillofacial Surgery Service, Lima-Peru.

Keywords: Odontoma; Mandibular neoplasms (source: MeSH NLM).

\section{Introducción}

Actualmente, la Organización Mundial de la Salud (OMS) ha clasificado al odontoma dentro de los tumores odontogénicos constituidos por epitelio y ectomesénquima odontogénico con o sin la presencia de mineralización de tejidos dentales ${ }^{1}$.

Pierre Paul Broca en 1867, fue quien introdujo el término odontoma, en mención a cualquier tumor originado por un desarrollo desmesurado, temporal o completo de tejidos dentales ${ }^{2}$.

Después en 1946 Thoma y Goldman enunciaron una clasificación de los odontomas, actualmente en desuso 3. La clasificación de los odontomas se dan teniendo en cuenta la disposición y el grado de variación de las células odontogénicas, en dos formas: compuestos (OCp) y complejos (OC) ${ }^{1,4}$. El OCp describe una morfodiferenciación e histodiferenciación, mientras el OC únicamente describe histodiferenciación ${ }^{5}$. En el OCp se forman diversas estructuras dentales de diferentes formas (dentículos), en tanto que en el OC se forma una masa sólida de tejidos dentales duros y blandos distribuidos de manera desorganizada sin parecerse a la morfología de un diente $4,6,7$.

Los odontomas son hamartomas benignos, no agresivos, que están compuestos por esmalte, dentina, cemento y tejido pulpar ${ }^{8}$; resultan del crecimiento de células epiteliales y mesenquimatosas diferenciadas, en donde los ameloblastos y odontoblastos forman esmalte y dentina que se depositan de manera anormal o defectuosa ${ }^{9,10}$. La etiología de los odontomas es desconocida y en ella se le implican diferentes factores como los traumatismos, las infecciones, mutaciones genéticas, hiperactividad odontoblástica o las alteraciones en el gen de control del desarrollo dentario. La mayor parte de estos tumores se descubren durante la segunda y tercera década de la vida y no tienen una clara predilección por el sexo ${ }^{11-14}$.

Esta patología se visualiza como una masa amorfa, mineralizada, asintomática, radiográficamente se observa una imagen radiopaca, rodeada de un halo radiolúcido de bordes regulares ${ }^{14}$. Los odontomas compuestos se localizan en la región anterior del maxilar superior mientras que los odontomas complejos se ubican en la región posterior de la mandíbula ${ }^{15,16}$.

En algunos casos los odontomas se asocian con síndromes, tales como el síndrome de Gardner ${ }^{16,17}$. Los odontomas, son considerados hamartomas más que verdaderas neoplasias, debido a que tienen su origen en proliferaciones accesorias del epitelio odontogénico en la cavidad oral, éstos han sido ampliamente reportados en la literatura por ser el tumor odontogénico más común en los maxilares ${ }^{13}$.

\section{Reporte del caso}

Paciente varón de 37 años, natural y procedente de Lima - Perú, sin antecedentes sistémicos. Refiere que hace 15 días nota un aumento de volumen en región geniana izquierda, con fístula activa en esta misma región, asociado a dolor y secreción purulenta, por lo que decide visitar a odontólogo particular donde solicitan radiografía panorámica; a la evaluación de la misma, sugieren la impactación de la pieza 38 y una alteración patológica en este mismo lugar, recibiendo la recomendación de visitar a especialista, por lo que acude al servicio de cirugía bucal y maxilofacial del Hospital Nacional Hipólito Unanue para el diagnóstico y tratamiento de su afección.

Al examen clínico extraoral se evidencia leve tumefacción de hemicara izquierda de consistencia dura dolorosa a la palpación, con fistula activa y secreción purulenta. A la evaluación intraoral se evidencia fistula activa a nivel de la pieza 3.6 ausencia de piezas 3.7 y 3.8 , tumefacción que compromete fondo de surco vestibular de tercer cuadrante que se extiende desde la pieza 3.7 hasta el espacio retromolar izquierdo de $5 \times 3 \mathrm{~cm}$ aproximadamente de consistencia dura, con expansión de tabla lingual y vestibular dolorosa a la palpación con presencia de indentaciones sobre la mucosa del reborde alveolar de las pieza 3.7 y 3.8 (Figuras 1 y 2 ).

El examen radiográfico mostró una gran masa calcificada, de bordes irregulares que se extendía desde distal de la primera molar mandibular izquierda hacia la rama mandibular, el tercer molar se localizó por debajo de la masa calcificada muy cerca de la basal mandibular izquierda (Figura 3A). La tomografía Cone Beam mostró adelgazamiento de las corticales linguales y bucales, con desplazamiento del nervio dentario inferior (Figura 3 B, C y D).

Se realiza la biopsia incisional enviándose la muestra a anatomía patológica. El resultado de la evaluación microscópica muestra una masa calcificada que contiene múltiples espacios ocupados por tejido blando, la estructura de la masa calcificada tiene características de dentina, osteodentina, cemento y osteocemento, en los espacios de tejido blando se distingue matriz de esmalte e islas de epitelio odontogénico rodeada por tejido inflamatorio, estas características microscópicas son contributorias para odontoma complejo infectado.

El control de la infección se realizó con clindamicina $300 \mathrm{mg}$ vía oral cada 8 horas por 7 días y ciprofloxacino 500 mg vía oral cada 12 horas por 7 días; la disminución 


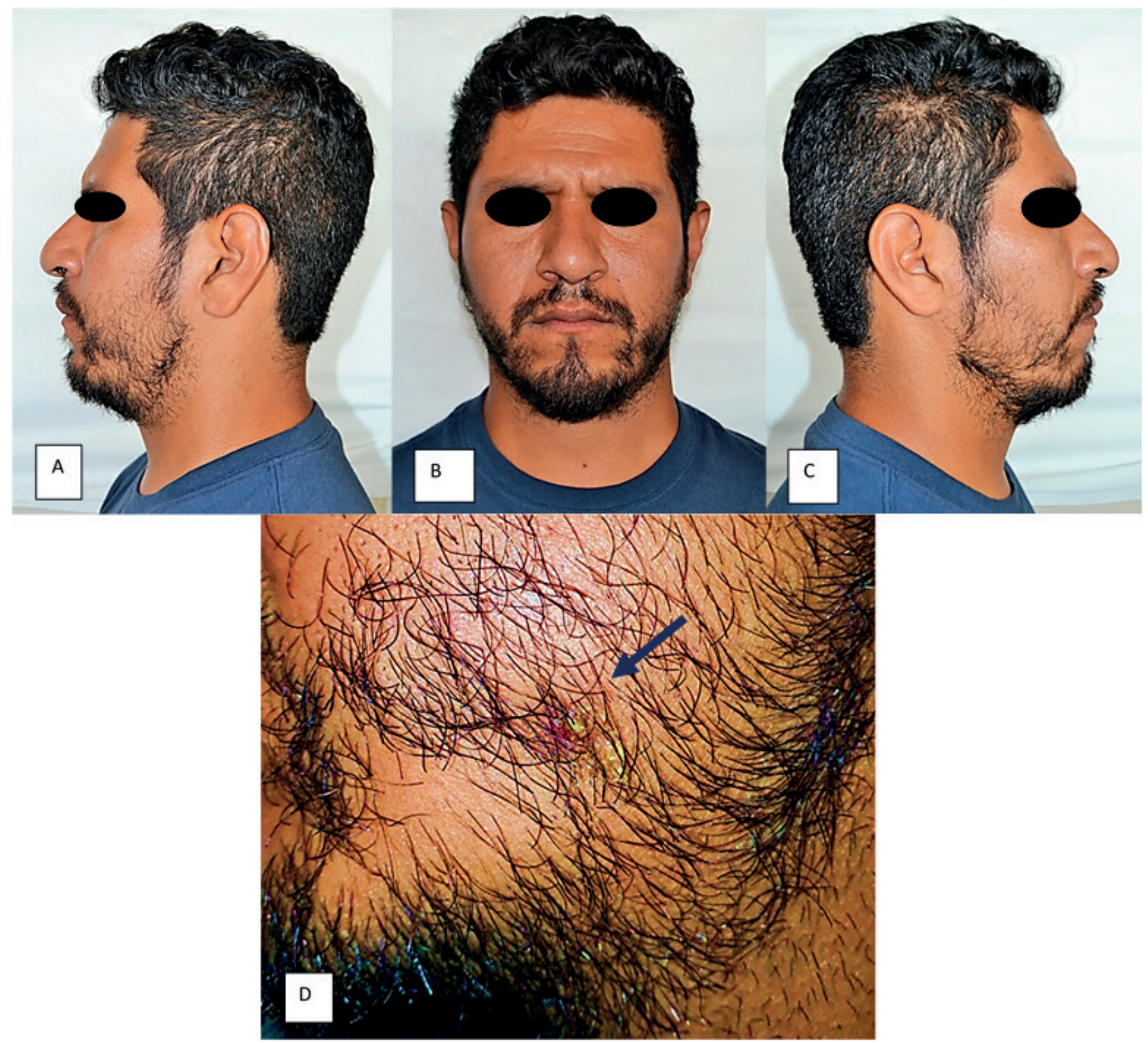

Figura 1. A, B y C. Examen extraoral que evidencia aumento de volumen en región geniana izquierda. D. Imagen extraoral que muestra fístula extraoral con secreción purulenta
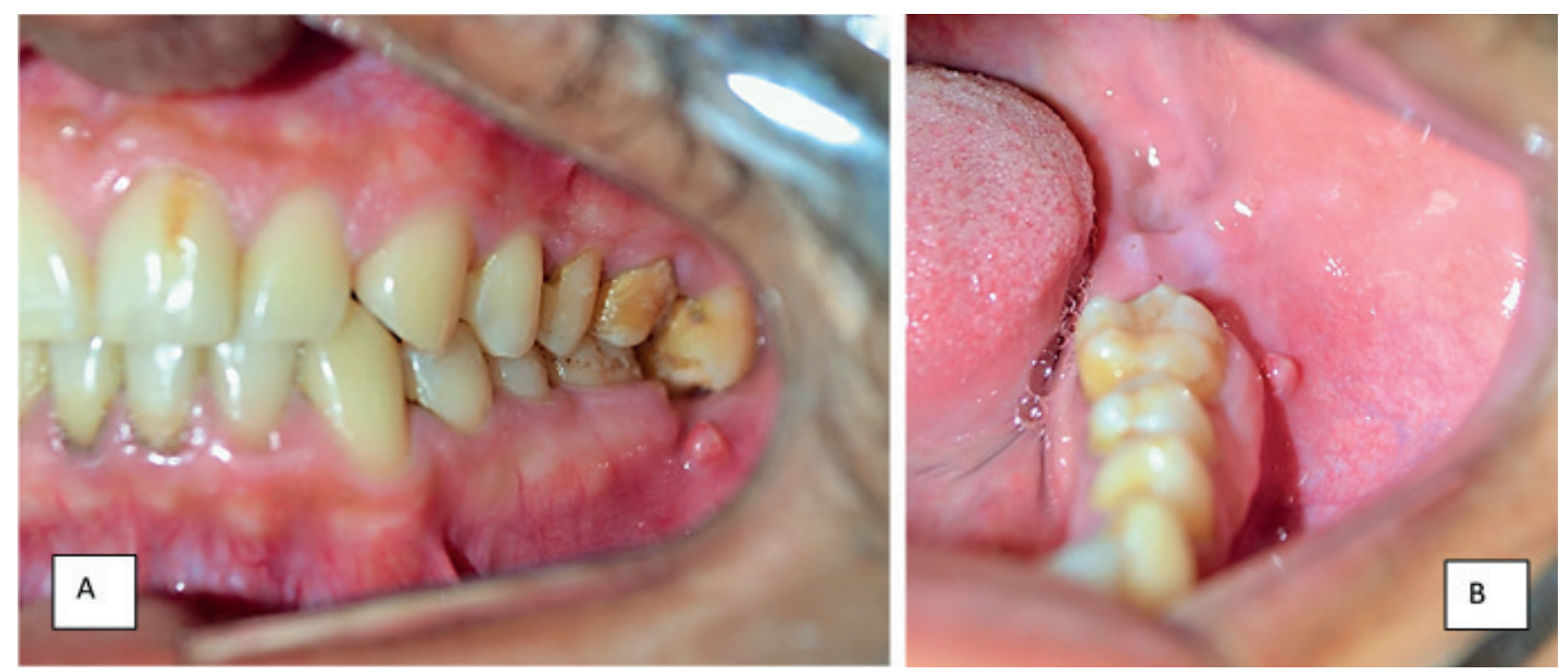

Figura 2. A y B. Examen intraoral que evidencia aumento de volumen de fondo de surco vestibular izquierdo que se extiende a reborde alveolar de piezas 3.7 y 3.8 donde se muestra fistula intraoral no activa a nivel de la pieza 3.6, con presencia de indentaciones en reborde alveolar de piezas 3.7 y 3.8 

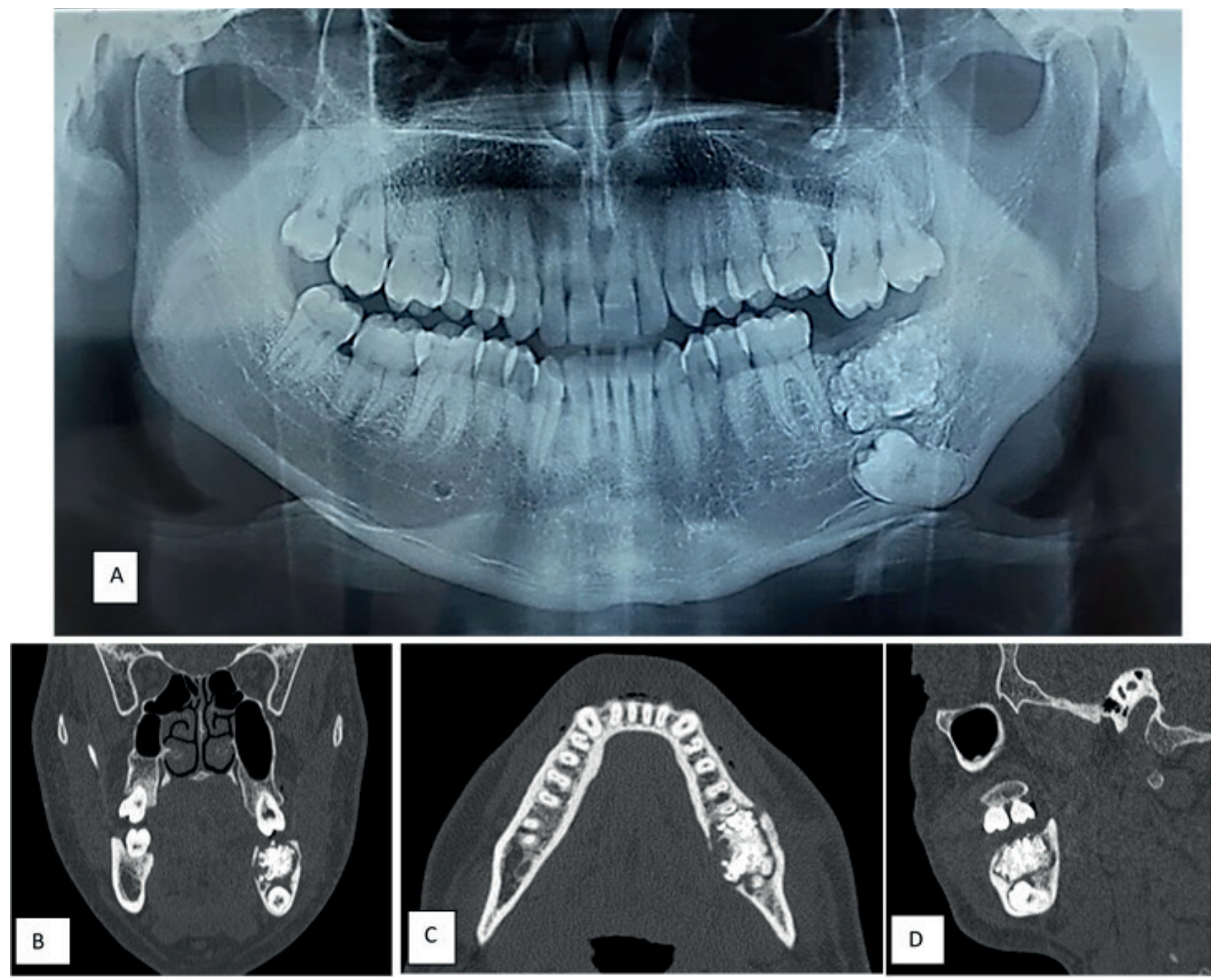

Figura 3. A. El examen radiográfico mostró una gran masa calcificada, de bordes irregulares que se extendía desde distal de la primera molar mandibular izquierda hacia la rama mandibular, el tercer molar se localizó por debajo de la masa calcificada muy cerca de la basal mandibular izquierda. B, C y D. La tomografía Cone Beam mostró adelgazamiento de las corticales linguales y bucales, con desplazamiento del nervio dentario inferior

de los síntomas se observó una semana después. Posteriormente el paciente fue programado para la enucleación del odontoma y exodoncia de la pieza 3.8, utilizando el abordaje de osteotomía sagital de rama mandibular (Figura 4) con bloqueo intermaxilar y cerclaje tipo Ivy por 15 días, Se decide realizar el bloqueo intermaxilar por la pérdida de volumen óseo y la escasa continuidad de los segmentos, evitando de esta manera fuerzas mandibulares inadecuadas después del procedimiento, así mismo la oclusión del paciente se mantuvo estable.

El paciente permaneció hospitalizado y teniendo un postoperatorio sin complicaciones fue dado de alta dos días después.

Una radiografía panorámica postoperatoria mostró una adecuada fijación del material de osteosíntesis conservando la basal mandibular, el paciente presentó clínicamente una oclusión estable y una resolución completa de la fístula extraoral que presentaba al momento de la consulta.

\section{Discusión}

Los odontomas son tumores benignos que se presentan con frecuencia en la cavidad oral, en la mayoría de los casos no dan ningún tipo de sintomatología, el signo más común es el retraso de la erupción dentaria permanente ${ }^{18}$. Las radiografías periapicales y panorámicas son ayudas diagnósticas que en primera medida son esenciales ${ }^{19}$.

La frecuencia reportada de tumores odontogénicos en el mundo varía con un amplio rango $(1-32 \%)^{20}$.

Los odontomas constituyen el grupo de tumoraciones benignas más frecuentes $(20-40 \%)$ dentro de los tumores odontogénicos ${ }^{21}$.

En la literatura se reconocen tres presentaciones clínicas de los odontomas: intraóseos, extraóseos y erupcionados, siendo los intraóseos los más frecuentes ${ }^{22}$.

La localización más comúnmente reportada es el área incisivo-canina del maxilar superior (67\%), seguida por las zonas anteroinferior y posteroinferior de la mandíbula $(33 \%)^{20-22}$.

Los odontomas compuestos se presentan claramente con mayor frecuencia que los odontomas complejos ${ }^{23}$. El odontoma compuesto aparece con mayor frecuencia en la parte anterior del maxilar, sobre la corona de un 


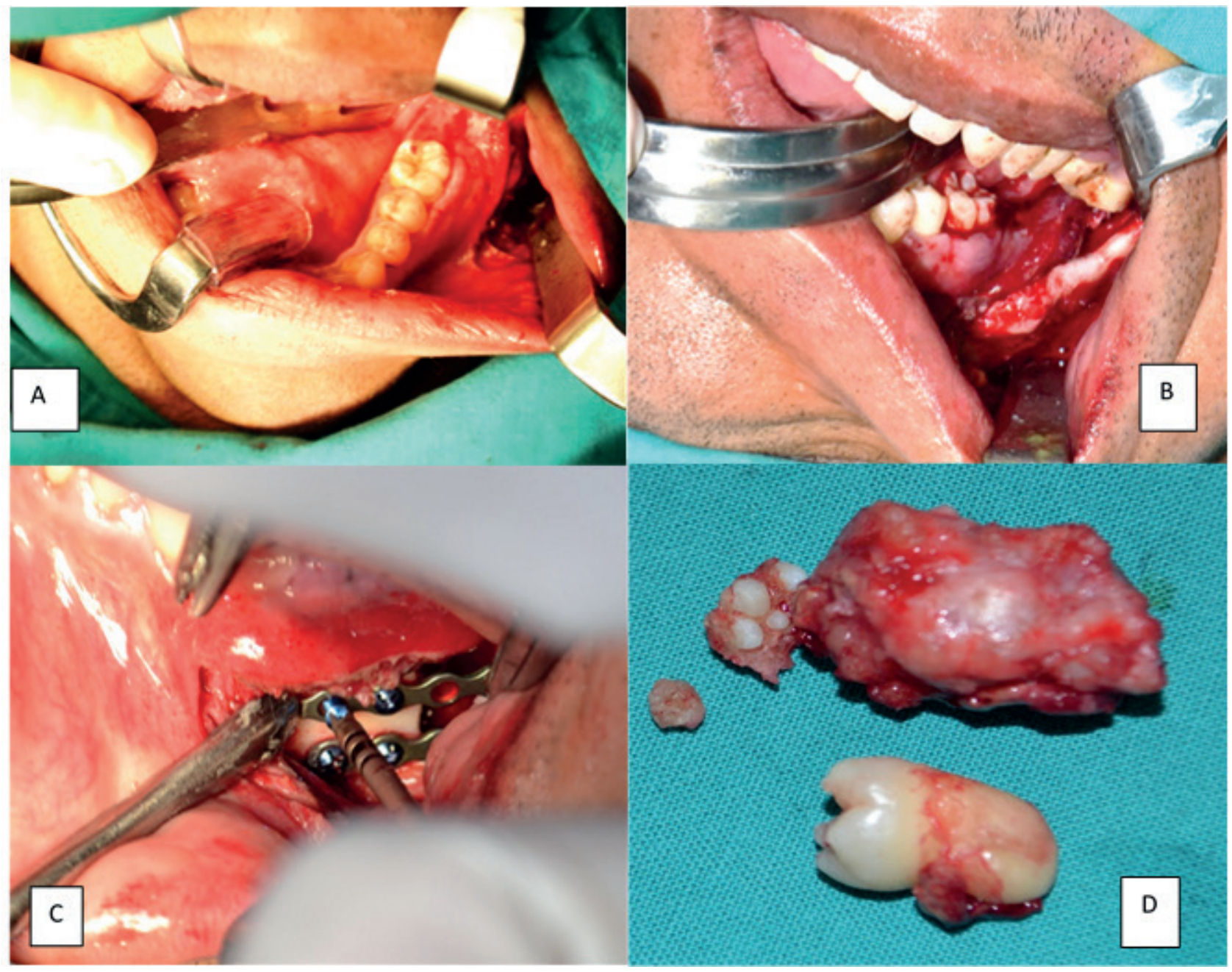

Figura 4. A, B, C. Imágenes intraorales donde se evidencia el abordaje quirúrgico sagital de rama unilateral. D. Odontoma complejo con la pieza 3.8

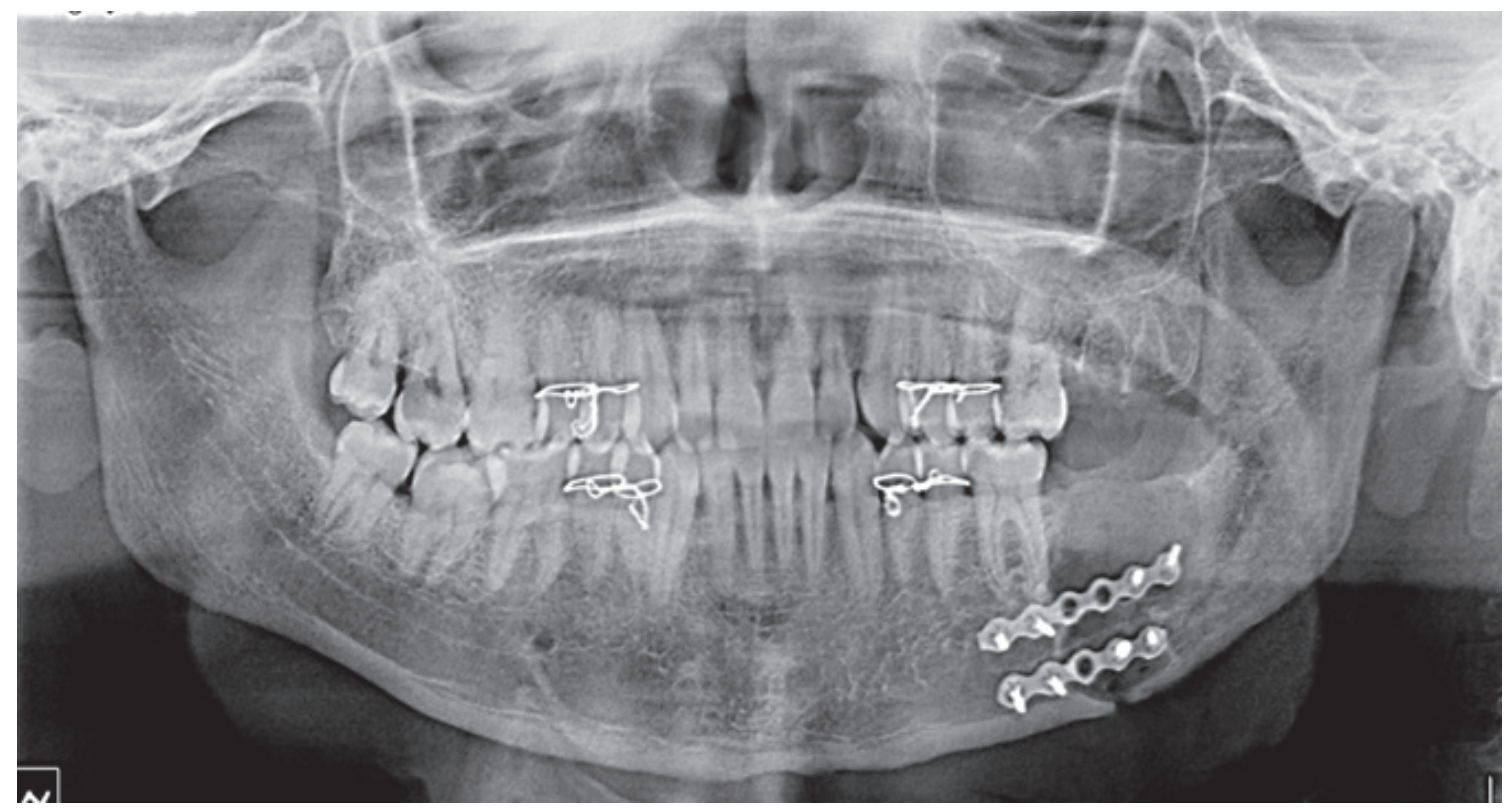

Figura 5. Imagen radiológica posterior a la cirugía, se evidencia material de osteosíntesis en posición con cerclaje tipo Ivy a nivel de premolares derecha e izquierda donde la continuidad de la basal mandibular del lado afectado está conservada 
diente(s) no erupcionado(s) o entre las raíces de dientes erupcionados $(61 \%)$ mientras que el complejo lo hace en la parte posterior de la mandíbula (59\%) ${ }^{26-28}$.

Radiográficamente los odontomas compuestos se observan como masas radiopacas de márgenes irregulares que adoptan una configuración similar a los dientes, con un borde periférico radiolúcido, mientras los complejos muestran una radiopacidad única ${ }^{4}$.

Histológicamente los odontomas compuestos se caracterizan por la presencia de tejidos dentales, esmalte desmineralizado, dentina, cemento y pulpa, dispuestos en forma organizada de estructuras dentales y rodeados parcialmente por una cápsula de tejido conectivo, en cambio los odontomas complejos se observa una masa desorganizada de tejidos dentales duros ${ }^{6}$.

El tratamiento aceptado de los odontomas es la exéresis; sin embargo, siempre que el tamaño y la ubicación del odontoma puedan poner en peligro el nervio de la mandíbula inferior o los dientes adyacentes, debe considerarse un enfoque diferente para evitar cualquier problema. Los odontomas complejos grandes en la mandíbula son raros; así, hay poca información disponible sobre el abordaje quirúrgico preferido para estas lesiones ${ }^{26-33}$.

Las ventajas de una osteotomía sagital de rama para obtener acceso para la extirpación de una lesión benigna grande de la mandíbula en una persona joven superan a los de la técnica de resección en bloque convencional. Evita la formación de un gran defecto en el hueso cortical con su mayor riesgo asociado de fractura ${ }^{34}$.

Se logra un excelente acceso a la lesión, especialmente cuando la lesión se encuentra completamente dentro de las placas corticales, y la continuidad de la mandíbula se conserva después de la fijación de los fragmentos proximal y distal. La capacidad de disecar y preservar el paquete neurovascular, especialmente cuando ha sido desplazada por una lesión, es una ventaja decidida sobre otras técnicas de resección donde el nervio probablemente tendría que ser sacrificado ${ }^{35}$.

Es de conocimiento, que el tratamiento aceptado de los odontomas es la enucleación.

Sin embargo, siempre que el tamaño y la ubicación del odontoma puedan poner en peligro al nervio dentario inferior, a los dientes adyacentes y/o ocasionar una fractura patológica de la mandíbula se deberían optar por otras alternativas de tratamiento, que además nos permita conservar tejido óseo intacto y una de ellas es la osteotomía sagital de rama siendo mucho más ventajoso su uso cuando el tumor se encuentra dentro de las dos tablas (lingual y vestibular) evitando entonces de esta manera fracturas patológicas que complicarían la cirugía en un abordaje convencional como es la enucleación.

\section{Referencias bibliográficas}

1. Barnes L, Eveson JW, Relchart P et al. Pathology and genetics of head and neck tumors. WHO. Classification of tumors. Lyon: IARC Press; 2005. pp. 284-327.
2. Philipsen HP, Reichart PA. Classification of odontogenic tumours. A historical review. J Oral Pathol Med. 2006;35(9):525-529.

3. Thoma KM, Goldman HM. Oral pathology. 5th ed. St Louis: The CV Mosby Company; 1960. pp. 1221-1222.

4. Soluk-Tekkesin M, Pehlivan S, Olgac V, Aksakall N, Alatli C. Clinical and histopathological investigation of odontomas: review of the literature and presentation of 160 cases. J Oral Maxillofac Surg. 2012;70(6):13581361.

5. Sánchez A, Liceaga CJ. Odontoma complejo de tamaño inusual. Revisión de literatura y reporte de un caso. Rev Mex Cir Bucal Max. 2012;8(3):103-109.

6. Vázquez DJ, Gandini PC, Carbajal EE. Odontoma compuesto: diagnóstico radiográfico y tratamiento quirúrgico de un caso clínico. Av. Odontoestomatol. 2008;24(5):307-312.

7. Yépez J, Castillo JL, Molina M. Odontoma compuesto mandibular. Reporte de caso. Revista Medula. 2009;18:138-143.

8. Raval N, Mehta D, Vachhrajani K, Nimavat A. Erupted odontoma: a case report. J Clin Diagn Res. 2014;8(7):ZD10-ZD11.

9. Padmanabhan MY, Pandey RK, Aparna R. Erupted composite odontoma associated with malformed teeth unusual dental aberrations following maxillofacial trauma. Rom J Morphol Embryol. 2013;54(4):1153-1156.

10. Sreedharan S, Krishnan IS. Compound odontoma associated with impacted maxillary incisors. J Indian Soc Pedod Prev Dent. 2012;30(3):275-278.

11. Yadav M, Godge P, Meghana SM, Kulkarni SR. Compound odontoma. Contemp Clin Dent. 2012;3(Suppl 1):S13-S15.

12. Bagewadi SB, Kukreja R, Suma GN, Yadav B, Sharma H. Unusually large erupted complex odontoma: A rare case report. Imaging Sci Dent. 2015;45(1):49-54.

13. Nelson-Filho P, Silva RA, Faria G, Freitas AC. Odontomalike malformation in a permanent maxillary central incisor Sub sequent to trauma to the incisor predecessor. Dent Traumatol. 2005;21(5):309-312.

14. Chang JY, Wang JT, Wang YP, Liu BY, Sun A, Chiang CP. Odontoma: a clinicopathologic study of 81 cases. J Formos Med Assoc. 2003;102(12):876-882.

15. Ballesteros G, Goenaga B, Toro F, Villa L, Arango M. Odontoma compuesto maxilar, reporte de un caso y revisión de literatura. Acta Odontol Venez. 2011;49(2):1-11.

16. Sood PB, Patil B, Godhi S, Shetty DC. Multiple supernumerary teeth and odontoma in the maxilla: A case report. Contemp Clin Dent. 2010;1(1):45-46.

17. Hisatomi M, Asaumi JI, Konouchi H, Honda Y, Wakasa T, Kishi K. A case of complex odontoma associated with an impacted lower deciduous second molar and analysis of the 107 odontomas. Oral Dis. 2002;8(2):100-105.

18. Tirado-Amador LR, González-Martínez FD, Sir-Mendoza FJ. Uso controlado de los rayos $\mathrm{X}$ en la práctica odontológica. Rev Cienc Salud. 2015;13(1):99-112. DOI: 10.12804/ revsalud13.01.2015.08. 
19. An SY, An CH, Choi KS. Odontoma: a retrospective study of 73 cases. Imaging Sci Dent. 2012;42(2):77-81.

20. Buchner A, Merrell PW, Carpenter WM. Relative frequency of centralodontogenic tumors: a study of 1,088 cases from Northern California and comparison to studies from other parts of the world. J Oral Maxillofac Surg. 2006;64(9):1343-1352.

21. Hidalgo Sánchez O, Leco Berrocal MI, Martínez-González JM. Meta-análisis sobre la epidemiología y clínica de los odontomas. Med Oral Patol Oral Cir Bucal. 2008;13(5):306-310.

22. Junquera L, de Vicente JC, Roig P, Olay S, Rodríguez-Recio O. Intraosseous odontoma erupted into the oral cavity: an unusual pathology. Med Oral Patol Oral Cir Bucal. 2005;10(3):248-251.

23. Mosqueda-Taylor A, Ledesma-Montes C, Caballero-Sandoval S, Portilla-Robertson J, Ruíz-Godoy Rivera LM, Meneses-García A. Odontogenic tumors in Mexico: a collaborative retrospective study of 349 cases. Oral Surg Oral Med Oral Pathol Oral Radiol Endod. 1997;84(6): 672-675.

24. Amado-Cuesta S, Gargallo-Albiol J, Berini-Aytés L, Gay-Escoda C. Review of 61 cases of odontoma. Presentation of an erupted complex odontoma. Med Oral. 2000;8(5):366-373.

25. Fernández AM, Duarte EC, Pimenta FJ, Souza LN, Santos VR, Mesquita RA et al. Odontogenic tumors: a study of 340 cases in a Brazilian population. J Oral Pathol Med. 2005;34(10):583- 587.

26. Hidalgo O, Leco MI, Martínez JM. Metaanalysis of the epidemiology and clinical manifestations of odontomas. Med Oral Patol Oral Cir Bucal. 2008;13(11):730-734.

27. Maden EA, Altun C, Sencimen M. A case report of compound odontoma associated with an unerupted maxillary central incisor. Arch Clin Exp Surg. 2014;3(1):47-51.
28. Bodin I, Julin P, Thomsson M. Odontomas and their pathological sequels. Dentomaxillofac Radiol. 1983;12(2):109-114.

29. Frame JW. Surgical excision of a large complex composite odontome of the mandible. Br J Oral Maxillofac Surg 1986;24(1):47-51.

30. Goldberg H, Schofield IDF, Popovich ID. Cystic complex composite odontoma. Report of two cases. Oral Surg. 1981;51(1):16-20.

31. Laskin DM. Discussion-Surgical management of a large, complex mandibular odontoma by unilateral sagittal split osteotomy. J Oral Maxillofac Surg. 1989;47(2):179-84.

32. Shteyer A, Taicher S, Marmary Y. Odontoma in the subcondylar region. Br J Oral Surg. 1979;17(2):161-5

33. Wong GB. Surgical management of a large, complex mandibular odontoma by unilateral sagittal split osteotomy. J Oral Maxillofac Surg.1989;47(2):179-84.

34. Frame JW. Surgical excision of a large complex composite odontome of the mandible. Br J Oral Maxillofac Surg. 1986;24(1):47-51.

35. Barnard D. Surgical access to a complex composite odontome by sagittal splitting of the mandible. Br J Oral Surg. 1983;21(1):44-8.

36. Obando-Pereda G, Torres-Chavez K, Salas-Beltran H, Hofling JF. Análisis de la composición química, capacidad de sellado apical y propiedades antimicrobianas del MTA y del cemento portland. Endo. 2009;27(3):111120.

37. Borrás AC, Catalá PM, Estrela SF. Utilización del MTA en el tratamiento de situaciones complejas de inmadurez radicular. Odontol Pediatr. 2006;14(3):76-81.

38. Duarte MA, Marciano MA, Vivan RR, Tanomaru FM, Tanomaru JM, Camilleri J. Tricalcium silicate-based cements: properties and modifications. Braz Oral Res. 2018;32(1):111-118. 
